Artigo

\title{
Utilização de Técnicas de Inteligência Computacional na Predição de Dados Meteorológicos
}

\author{
Eluã Ramos Coutinho, Robson Mariano Silva, Angel Ramon Sanchez Delgado \\ Departamento de Matemática, Universidade Federal Rural do Rio de Janeiro, \\ Seropédica, RJ, Brasil
}

Recebido: 22/11/2013 - Aceito: 29/5/2015

\begin{abstract}
Resumo
O presente trabalho apresenta uma abordagem computacional para a predição de um passo à frente em séries de dados meteorológicos pertencentes às regiões de Paty do Alferes e Paracambi, situadas no estado do Rio de Janeiro (RJ). Para tanto, foram utilizados dois modelos de Redes Neurais Artificiais (RNAs): Perceptrons de Múltiplas Camadas (MLP) e Função de Base Radial (RBF). Para confirmar o desempenho dos modelos foi realizada a predição de variáveis horárias e mensais, que foram comparadas com resultados obtidos por modelos de Regressão Linear Múltipla (RLM), confrontadas com os dados registrados pelas estações meteorológicas e analisadas por meio de técnicas estatísticas, apresentando resultados favoráveis entre $91 \%$ a $96 \%$ de acerto para todos os casos. Além disso, as previsões também demonstraram uma forte correlação linear com os dados registrados, mantendo-se entre 0,61 a 0,94 . Como resultado, pode se destacar as RNAs como uma forte ferramenta para predição dos dados meteorológicos analisados.

Palavras chave: séries de dados meteorológicos, passo à frente, redes neurais artificiais.
\end{abstract}

\section{Using Computational Intelligence Technique for the Meteorological Data Prediction}

\begin{abstract}
This paper presents a computational approach for the one step ahead prediction in meteorological data series belonging to regions of Paty do Alferes and Paracambi, both located in the state of Rio de Janeiro (RJ). Two models of Artificial Neural Networks (ANN) were used: Multilayer Perceptron (MLP) and Radial Basis Function (RBF). To confirm the performance of the models were performed the prediction of hourly and monthly variables. These data were compared with results obtained by Multiple Linear Regression models (RLM), but also with the data registered by meteorological stations and analyzed by statistical techniques. It showed a favorable result, reaching between $91 \%$ and $96 \%$ of correct predictions for all cases. Moreover, the predictions also showed a strong linear correlation with the actual data, keeping it from 0.61 to 0.94 . As a result, the RNAs can stand out as a strong tool for prediction of meteorological data analyzed.

Keywords: series of meteorological data, step ahead, artificial neural networks.
\end{abstract}

\section{Introdução}

Prever informações sobre o clima em período de tempo hábil é uma tarefa complexa, que pode depender de informações de satélites, análise de dados históricos, estudo sobre as marés, nuvens e outros. No entanto, pesquisas ocorridas durante anos têm demonstrado que em muitas regiões do mundo, o clima sazonal é potencialmente previsível (Goddard et al., 2001).

Contudo, Palmer e Anderson (1994) destacam que mudanças significativas são produzidas nas probabilidades de diferentes regimes climáticos que ocorrem ao longo de uma temporada, quando as condições de contorno que forçam a atmosfera como temperaturas da superfície do mar - TSM, e características da superfície da terra, estão fortemente perturbadas. Isto faz com que as previsões utilizáveis sejam possíveis sob certas condições. Na medida em que as condições de contorno relevantes e seus impactos climáticos associados são previsíveis, predições sazonais hábeis podem ser realizadas. 
Sabe-se que determinadas condições climáticas podem favorecer ou prejudicar diretamente diferentes setores da economia. Segundo dados do CEPEA (2013), a agricultura foi responsável por $23,82 \%$ do produto interno bruto (PIB) no ano de 2011. Também neste ano, catástrofes relacionadas ao excesso de chuvas foram responsáveis por aproximadamente 1000 mortes na região serrana do estado do Rio de Janeiro, e, no inicio de 2012, deixaram mais de 40.000 desabrigados e mais de 100 cidades em estado de emergência na região sudeste do país (CEPED, 2014).

Por isso, prever essas informações podem auxiliar instituições públicas e privadas a traçar planos para maximização de sua produção ou mesmo salvaguardar a vida e o patrimônio publico. Para tanto, vários modelos tem sido comumente aplicados na predição de variáveis meteorológicas como as interpolações e as regressões.

Gois et al. (2005) aplicaram regressão linear (RL) para estimar as médias dos dados de temperatura máxima e mínima do ar mensais de regiões do estado de Alagoas, e, junto a outras variáveis, foi possível identificar áreas susceptíveis a desertificação.

Em 2008, a regressão linear (RL) foi utilizada para calcular a equação de Angstrom-Prescott e estimar a radiação solar global de 11 estações meteorológicas na Venezuela, fazendo uso de dados da luz solar (Almorox et al., 2008).

Também em 2008, na Nigéria, regressões lineares múltiplas foram aplicadas em diferentes parâmetros meteorológicos para prever a relação entre as radiações solares globais (Falayi et al., 2008). Já no ano de 2011, o modelo de regressão linear foi utilizado para estimar médias dos dados de temperatura diurna, noturna e diária de São Joaquim em Santa Catarina (Vieira et al., 2012).

Entretanto, esses modelos podem depender de um grande número de dados históricos e de dados de regiões vizinhas, e mesmo assim alguns ignoram a variação espacial local das variáveis em estudo, o que pode acabar gerando erros grotescos devido a baixa precisão apresentada em algumas estimativas (Rolim et al., 2010).

Estes fatores incentivam a busca por metodologias que possam aprimorar o processo de predição e gerar dados consideravelmente próximos dos valores reais. Isto tem possibilitado o emprego de técnicas de inteligência computacional conhecidas como redes neurais artificiais (RNA).

As RNAs são sistemas computacionais de processamento paralelo com capacidade de armazenar conhecimento empírico. Segundo Haykin (2001), as RNAs se assemelham ao cérebro humano por dois fatores: o conhecimento e os pesos. Sendo assim, o conhecimento é adquirido através da aprendizagem, e os pesos, que são a intensidade das conexões entre os neurônios, é utilizado para armazenar estes conhecimentos.

Estas características fazem com que as RNAs sejam aplicadas em várias áreas e apresentem resultados bastante expressivos, tais como reconhecimento de padrões, contro- le de sistemas, otimização, processamento de sinais e predição. No que tange a área de predição de dados meteorológicos, a aplicação de modelos baseados em RNAs vem despertando o interesse de diversos pesquisadores.

Litta et al. (2013) aplicaram uma RNA na predição de tempestades na região de Kolkata na Índia. Maqsood et al. (2004) utilizaram RNAs para fornecer previsões de 24 horas de temperatura do ar, velocidade do vento e umidade relativa do ar no Aeroporto de Regina, no Canadá. Já Bustami et al. (2006) aplicou uma RNA na estimativa de dados de precipitação e na previsão do nível de água no rio Bedup, situado no estado de Sarawak, na Malásia. Todas essas aplicações apresentaram resultados satisfatórios com relação as medidas estatíticas utilizadas.

Sendo assim, o presente trabalho tem como objetivo aplicar e comparar modelos de RNAs de Perceptrons de Múltiplas Camadas (MLP) e Função de Base Radial (RBF) na predição de um passo a frente de séries de dados meteorológicos, obtidas junto as estações dos municípios de Paty do Alferes e Paracambi ambos situados no estado do Rio de Janeiro.

\section{Material e Métodos}

\subsection{Localidades de estudo}

As informações meteorológicas utilizadas neste estudo foram provenientes de duas estações situadas nos municípios de Paty do Alferes $\left(22,35^{\circ} \mathrm{S} ; 43,41^{\circ} \mathrm{W}\right.$ e $\left.507 \mathrm{~m}\right)$ e Paracambi $\left(22,62^{\circ} \mathrm{S} ; 43,72^{\circ} \mathrm{W}\right.$ e $\left.103 \mathrm{~m}\right)$, localizados no estado do Rio de janeiro (RJ), como pode ser visualizado na Figura 1.

O município de Paty do Alferes é conhecido por ser o principal produtor de tomates do estado, estando localizado na região Centro Sul Fluminense. Já Paracambi é um ponto estratégico localizado na região Metropolitana do estado, estando a $23 \mathrm{~km}$ de distância da Rodovia Raphael de Almeida Magalhães (Arco Metropolitano do Rio de Janeiro), e faz divisa com as Regiões Centro Sul Fluminense e com o Médio Paraíba. A cidade é cortada integralmente pelo Rio dos Macacos. Este passa pela área central do município, bem como seus afluentes e outros diversos canais que juntos desembocam no Ribeirão das Lages, um dos formadores da Bacia do Rio Guandu (Lemos et al., 2009).

\subsection{Dados utilizados}

O conjunto de dados utilizados neste estudo foram cedidos pelo Instituto Superior de Tecnologia de Paracambi (IST-Paracambi) e pelo Instituto Nacional de Meteorologia (INMET). Utilizou-se como variáveis: médias horárias de temperatura do ar, umidade relativa do ar e temperatura do solo pertencente ao município de Paracambi, além de médias mensais de temperatura máxima do ar e umidade relativa do ar pertencente ao município de Paty do Alferes. Os dados horários foram registrados por uma estação automática no período de 01/01/2011 a 


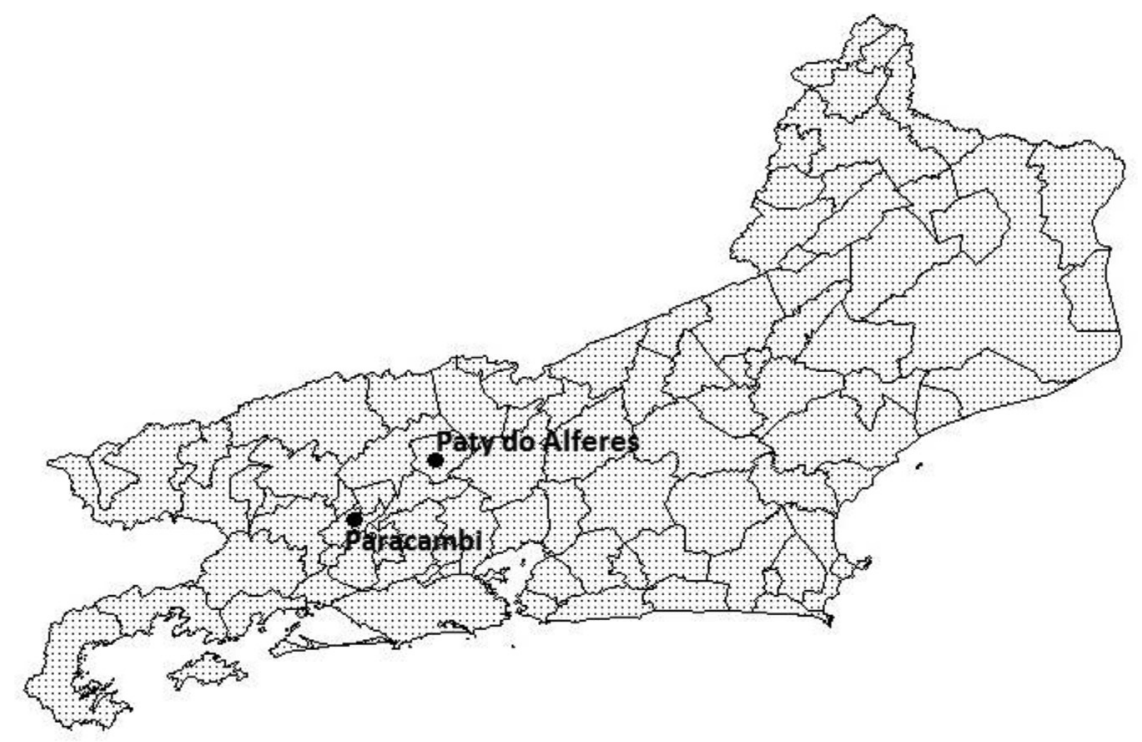

Figura 1 - Mapa do Rio de Janeiro com as regiões utilizadas no estudo: Paty do Alferes e Paracambi.

$31 / 01 / 2011$, enquanto os dados mensais foram registrados por uma estação convencional no período de 31/05/2002 a 31/12/2012. A escolha dessas variáveis se deu em função da série histórica de maior estabilidade.

\subsection{Redes Neurais Artificiais (RNAs)}

\subsubsection{Redes Perceptrons de Múltiplas Camadas (MLP)}

A RNA do tipo MLP pertence a classe de RNAs conhecida como feedfoward, além de ser uma aproximadora universal de funções, que tem sido aplicada em diferentes problemas combinatórios e na solução de diferentes tarefas como processamento de informações, reconhecimento de padrões, previsões de tempo, problemas de classificação, processamento de imagens, previsões de atividades sísmicas e outros (Shah e Ghazali, 2011).

A estrutura de uma MLP é formada por um conjunto de neurônios dispostos em camadas, contendo uma camada de entrada, uma ou mais camadas intermediárias, e uma camada de saída. Cada um dos neurônios da camada de entrada está conectada a todos os neurônios da camada intermediária. Da mesma forma, cada neurônio da camada intermediária está conectado com todos os neurônios da camada de saída (Hall et al., 1999; Asaduzzaman et al., 2010). A Figura 2 exibe a estrutura da MLP adotada no presente trabalho.

Entre cada uma das camadas existem pesos $w_{i}$, que estão associados às entradas $x_{i}$. Estes são responsáveis por realizar o produto $w_{i} x_{i}$, e dependendo do estímulo gerado pela soma ponderada das entradas pelos pesos, os neurônios ativam a função de transferência, que emite uma resposta para próxima camada. A camada de saída recebe os estímulos da camada intermediária e gera um padrão de resposta. Sendo assim, as camadas intermediárias funcionam como extratoras de características, onde seus pesos codificam as características dos padrões de entrada e permitem que a rede gere uma representação do problema mais detalhada e complexa (Santos e Filho, 2003).

Haykin (2001) ainda destaca que a RNA do tipo MLP possui neurônios capazes de gerar qualquer tipo de saída e que cada neurônio de uma MLP deve ser interpretado como modelo de um neurônio não linear (Figura 3 ), onde $x_{i}$ são os dados de entrada, $w_{i}$ são os pesos sinápticos, $b_{i}$ é o bias que tem o efeito de aumentar ou diminuir a entrada da função de ativação, $u_{i}$ é a combinação linear do $\sum_{i=1}^{n} w_{i j} x_{i}+b_{i}, f($.) é a função de ativação aplicada em $u_{i}$ e $y_{i}$ é a saída gerada pelo neurônio.

Existem muitos tipos de funções de ativação. Contudo, após a realização de vários testes com diferentes combinações de funções foi possível visualizar que as funções Logística e Tangente Hiperbólica Eqs. (1), (2) (Braga et al., 2012), apresentavam resultados mais apurados que as demais funções. Por isso, as mesmas foram adotadas neste estudo.

1) Função Logística:

$$
f\left(u_{i}\right)=\frac{1}{\left(1+e^{(-u i)}\right)}
$$

2) Função Tangente Hiperbólica:

$$
f\left(u_{i}\right)=\tanh \left(\frac{u_{i}}{2}\right)=\frac{1-e^{(-u i)}}{1+e^{(-u i)}}
$$

No entanto, a resposta de uma RNA ou resultado é obtido após o processo de treinamento, que realiza o ajuste dos pesos através de um algoritmo. O algoritmo de treinamento adotado neste estudo foi Back-propagation. 


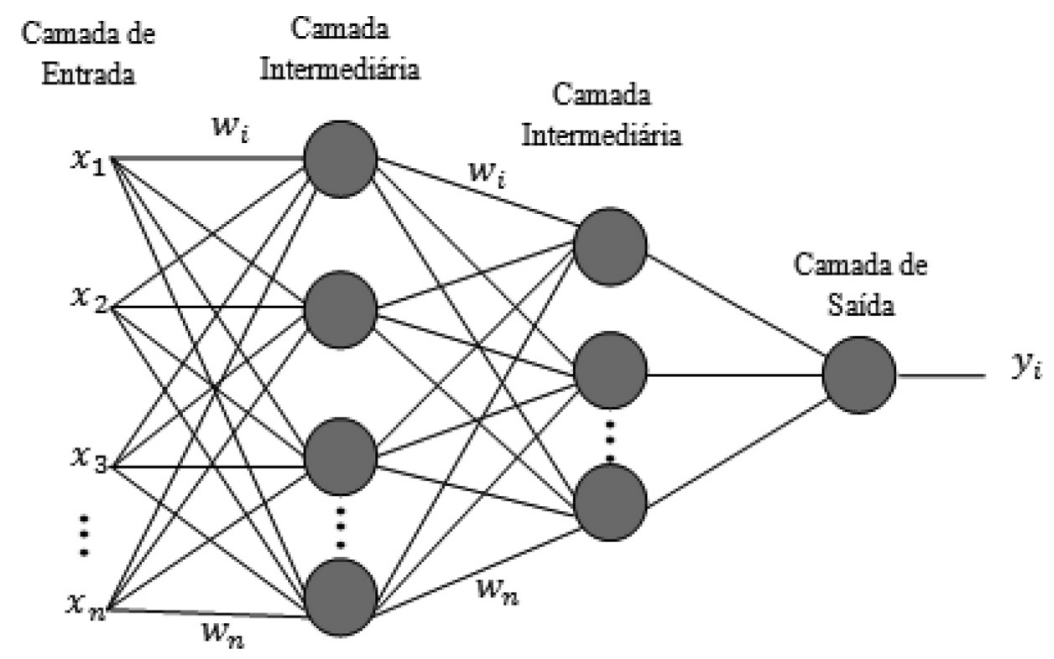

Figura 2 - Arquitetura da rede neural artificial MLP adotada neste estudo.

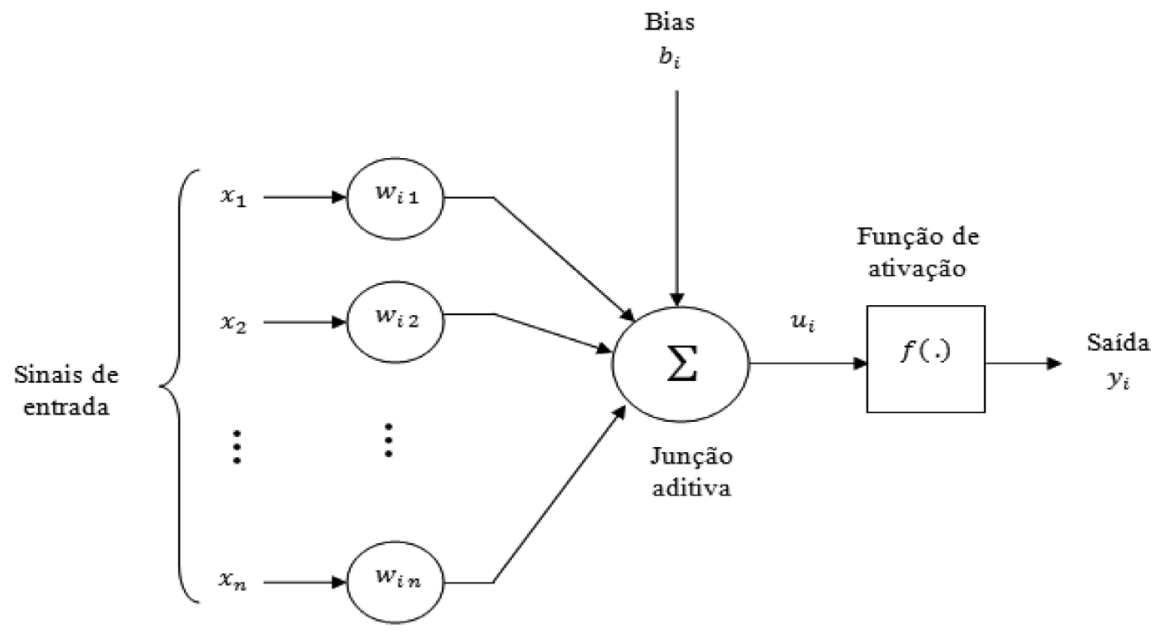

Figura 3 - Modelo de neurônio não linear . Fonte: adaptado de Haykin (2001).

\subsubsection{Back-propagation}

O algoritmo de Back-propagation ou algoritmo de retropropagação é uma técnica de treinamento supervisionada que realiza o ajuste dos pesos através do cálculo da diferença entre o valor predito pela rede e o valor esperado.

Este algoritmo é executado em duas fases definidas como forward e backward (Albarakati e Kecman, 2013). $\mathrm{Na}$ fase forward, as entradas são submetidas a rede e se propagam até a geração da saída, sem a alteração dos valores dos pesos. Na fase backward, calcula-se a diferença entre o valor predito $y_{p i}(t)$ pela rede e o esperado ou desejado $y_{d i}(t)$ Eq. (3) para que possa realizar o ajuste dos pesos a partir desta diferença, propagando-a até a camada de entrada.

$$
e(t)=y_{d i}(t)-y_{p i}(t)
$$

Este algoritmo é uma generalização da regra do delta. Dado um conjunto de treinamento, a cada passo $t$ deste treinamento é calculado o sinal de erro para um deter- minado neurônio $i$ da camada de saída de uma RNA do tipo MLP. Desta forma, a função de custo para uma RNA que tenha $n$ neurônios em sua camada de saída é definida como o somatório de $\frac{1}{2} e_{i}^{2}(t)$ (Guarnieri, 2006) Eq. (4).

$$
E(t)=\frac{1}{2} \sum_{i=1}^{n} e_{i}^{2}(t)
$$

A partir desta equação é calculado o ajuste dos pesos em direção contrária ao gradiente do erro. $\mathrm{O}$ ajuste de cada peso do neurônio da camada de saída é proporcional à derivada parcial de $E(t)$ em relação a cada um dos pesos $w_{i j}$ da camada de saída da rede. Assim é obtida a equação do ajuste de pesos de um neurônio $i$ qualquer da camada de saída Eqs. (5) e (6) (Boughrara et al., 2012).

$$
\frac{\partial E(t)}{\partial w_{i j}}(t)=-e_{i}(t) f^{\prime}\left(u_{i}(t)\right) h_{j}(t)
$$




$$
\Delta w_{i j}(t)=-\eta \frac{\partial E(t)}{\partial w_{i j}}
$$

onde $e_{i}(t)$ é o erro originado pela saída da rede, $f^{\prime}\left(u_{i}(t)\right)$ é a derivada da função aplicada nos valores ponderados pelos pesos do neurônio da camada de saída, $h_{j}(t)$ é o valor de entrada de uma conexão do neurônio da camada de saída, $\eta$ é a taxa de aprendizado do algoritmo de retropropagação que determina a velocidade de mudança dos pesos.

$\mathrm{Na}$ Eq. (6), o sinal negativo indica a descida do gradiente no espaço de pesos, ou seja, busca uma direção para minimização do $E(t)$. Esta equação pode ser reescrita como Eq. (7):

$$
\Delta w_{i j}(t)=-\eta \delta_{i}(t) h_{j}(t)
$$

Em que o gradiente local $\delta_{i}(t)$ é originado do produto do erro $e_{i}(t)$ pela derivada da função de ativação $f^{\prime}\left(u_{i}(t)\right)$ Eq. (8):

$$
\delta_{i}(t)=e_{i}(t) f^{\prime}\left(u_{i}(t)\right)
$$

Segundo Haykin (2001), quando um neurônio $i$ está localizado na camada oculta da rede, não existe uma resposta desejada para aquele neurônio. Desta maneira, um sinal de erro para o neurônio oculto deve ser determinado recursivamente em termos dos sinais de erro de todos os neurônios aos quais o neurônio oculto está diretamente conectado. Pode-se, então, redefinir o gradiente local $\delta_{i}(t)$ para o neurônio oculto $i$ por meio dos cálculos da derivada parcial $\partial E(t) / \partial y_{p i}(t)$ e aplicando a regra da cadeia para a derivada parcial de $\partial e_{k}(t) / \partial y_{p i}(t)$ até chegar a solução Eqs. (9), (10), (11), (12) e (13):

$$
\begin{aligned}
& \delta_{i}(t)=-\frac{\partial E(t)}{\partial y_{p i}(t)} \frac{\partial y_{p i}(t)}{\partial u_{i}(t)} \\
& =-\frac{\partial E(t)}{\partial y_{p i}(t)} f_{i}^{\prime}\left(u_{i}(t)\right) \\
& \frac{\partial E(t)}{\partial y_{p i}(t)}=-\sum_{k} e_{k}(t) f_{k}^{\prime}\left(u_{k}(t)\right) w_{k i}(t) \\
& =-\sum_{k} \delta_{k}(t) w_{k i}(t) \\
& \delta_{i}(t)=f_{i}^{\prime}\left(u_{i}(t)\right) \sum_{k} \delta_{k}(t) w_{k i}(t)
\end{aligned}
$$

No final, a atualização dos pesos da camada oculta é conseguido por meio da regra do delta Eq. (14).

$$
\Delta w_{i j}(t)=\eta \delta_{i}(t) x_{j}(t)
$$

onde $e_{k}(t)$ é o erro originado pela saída da rede, $f^{\prime}\left(u_{k}(t)\right)$ é a derivada da função aplicada nos valores ponderados pelos pesos do neurônio e as entradas da camada de saída, $w_{k i}(t)$ é o peso da ligação sináptica que conecta cada neurônio $k$ da camada seguinte ao neurônio $i, f^{\prime}\left(u_{i}(t)\right)$ é a derivada da função aplicada nos valores ponderados pelos pesos das sinapses do neurônio e as entradas da camada oculta, $\delta_{i}(t)$ é o gradiente local.

\subsubsection{Redes de Função de Base Radial (RBF)}

Assim como a rede MLP, a RBF é uma aproximadora universal de funções. No entanto, ela é ativada por meio da função da distância entre seus vetores de entrada e seus centros (Braga et al., 2012).

Em sua forma básica, a arquitetura de uma rede do tipo RBF é composta de três camadas, contendo uma camada de entrada, uma intermediária e uma de saída (Song e Kasabov, 2004). A Figura 4 demonstra a arquitetura adotada no trabalho.

A camada intermediária ou oculta de uma RBF utiliza funções de base radiais e tem a finalidade de agrupar os dados de entrada em clusters e transformar um conjunto de padrões de entrada não linearmente separáveis em um conjunto de saídas linearmente separáveis. A camada de saída tem a função de classificar os padrões recebidos da camada anterior através da combinação linear das saídas das funções (Braga et al., 2012).

Após testes e análises optou-se por utilizar a função de base radial gaussiana, que é uma das mais utilizadas na literatura Eq. (15).

$$
f(u)=e^{\left(-\frac{v^{2}}{2 \sigma^{2}}\right)}
$$

$\mathrm{Na}$ Eq. (15), $v=\left(\left\|x-t_{i}\right\|\right)$ é dado pela distância euclidiana, onde $x$ é o valor de entrada da rede, enquanto $t_{i}$ e $\sigma$ correspondem ao centro e a largura da função radial. Dessa maneira, a resolução de um determinado problema por uma RNA do tipo RBF consiste na resolução das funções (16) e (17) para chegar ao sistema Eq. (18).

$$
\begin{aligned}
& f(x)=\sum_{i=1}^{k} w_{j i} \varphi\left(\left\|x-t_{i}\right\|\right) \\
& y_{i}=\sum_{i=1}^{k} w_{j i} \phi\left(\left\|x-t_{i}\right\|\right)+w_{j 0}
\end{aligned}
$$

$$
\left[\begin{array}{cccc}
\varphi\left(\left\|x_{1}-t_{1}\right\|\right) & \ldots & \varphi\left(\left\|x_{1}-t_{2}\right\|\right) & \varphi\left(\left\|x_{1}-t_{N}\right\|\right) \\
\varphi\left(\left\|x_{2}-t_{1}\right\|\right) & \ldots & \varphi\left(\left\|x_{2}-t_{2}\right\|\right) & \varphi\left(\left\|x_{2}-t_{N}\right\|\right) \\
\vdots & \ddots & \vdots & \vdots \\
\varphi\left(\left\|x_{N}-t_{1}\right\|\right) & \ldots & \varphi\left(\left\|x_{N}-t_{2}\right\|\right) & \varphi\left(\left\|x_{N}-t_{N}\right\|\right)
\end{array}\right]\left[\begin{array}{c}
w_{1} \\
w_{2} \\
\vdots \\
w_{N}
\end{array}\right]=\left[\begin{array}{c}
y_{1} \\
y_{2} \\
\vdots \\
y_{N}
\end{array}\right]
$$

de base radial aplicadas nas entradas $x$ da RNA e dos seus

respectivos centros $t_{i}, w_{j 0}$ representa o bias, $\varphi\left(\left\|x-t_{i}\right\|\right)$ é o onde $w_{i j}$ são os pesos de cada conexão sináptica, $\phi$ é a matriz de interpolação originada do conjunto de $N$ funções 


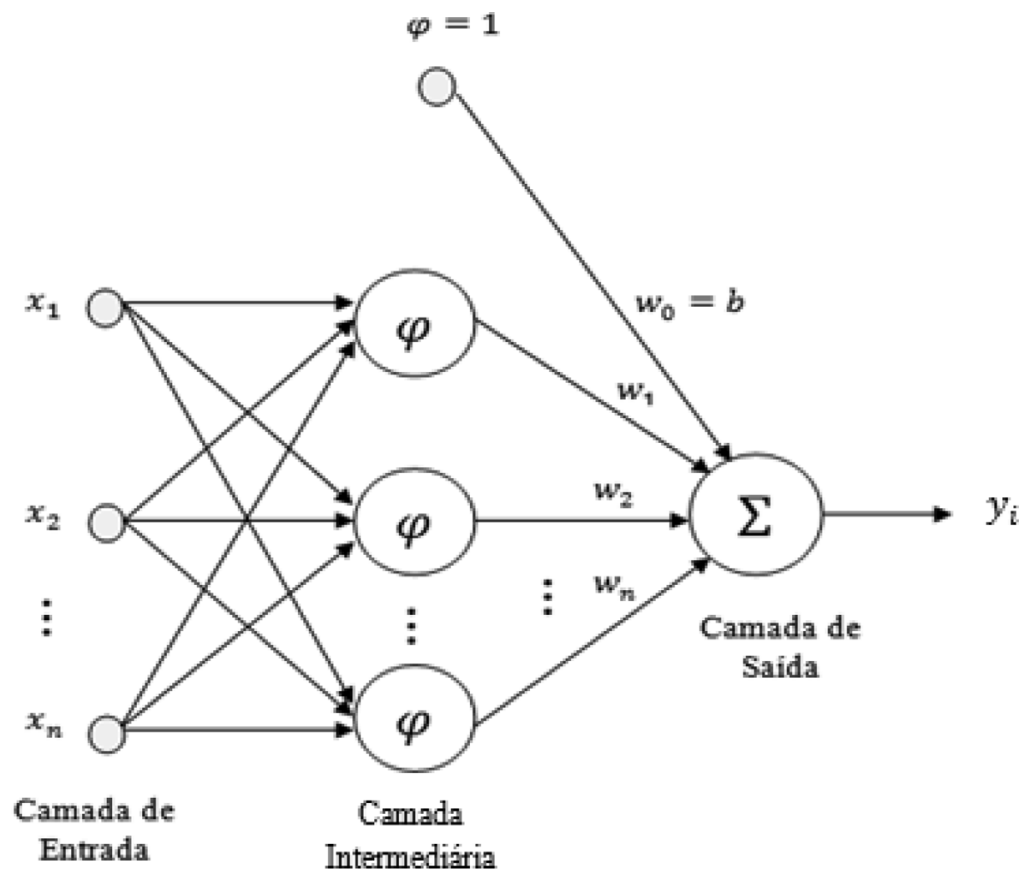

Figura 4 - Estrutura da RNA RBF adotada no trabalho.

conjunto de $N$ funções de base radial, ||.|| é a norma euclidiana, $y$ são as saídas geradas pela rede.

Assim como a RNA do tipo MLP, o resultado $S$ de uma RBF é conseguido através do ajuste dos pesos obtido por meio da minimização dos erros quadráticos, que comparam as saídas desejadas $y_{d i}$ com as saídas preditas $y_{p i}$ Eq. (19).

$$
S=\sum_{i=1}^{n}\left(y_{d i}-y_{p i}\right)^{2}
$$

Este ajuste é conseguido por meio do processo de treinamento, que em uma rede RBF pode acontecer de forma híbrida, tendo dois estágios. O primeiro estágio utiliza técnicas não supervisionadas como o algoritmo $K$ means-clustering, RNAs do tipo SOM e outros, para seleção dos centros das funções de base radiais. O segundo pode se utilizar de técnicas supervisionadas para definição dos pesos, como a Regra do Delta ou o Método de Decomposição em Valores Singulares (Hu e Hwang, 2002).

\subsection{Regressão Linear Múltipla (RLM)}

A regressão linear múltipla (RLM) é uma técnica que tem por finalidade analisar ou relacionar uma variável dependente a outras variáveis independentes também conhecidas como variáveis de previsão (Fonseca et al., 2012). Segundo Sousa et al. (2007), a relação entre uma variável dependente $Y$ e outras variáveis independentes $\left(X_{1}, X_{2}, X_{3}\right.$, ..., $X_{k}$ ), é formulada pelo seguinte modelo linear Eq. (20):

$$
Y_{i}=\alpha+\beta_{1} X_{1}+\beta_{2} X_{2}+\ldots+\beta_{K} X_{K}+U_{i}
$$

$\mathrm{Na}$ equação, $\alpha, \beta_{1}, \beta_{2}, \ldots, \beta_{k}$ são os coeficientes de regressão e $U_{i}$ são os distúrbios aleatórios ou erros aleatórios independentes (Sousa et al., 2007; Lyra et al., 2011).

O seguinte modelo ainda pode ser reescrito de forma matricial Eq. (21).

$$
\left(\begin{array}{l}
y_{1} \\
y_{2} \\
y_{3} \\
y_{4} \\
\ldots \\
y_{n}
\end{array}\right)=\left(\begin{array}{ccccc}
1 & x_{11} & x_{21} & \ldots & x_{k 1} \\
1 & x_{12} & x_{22} & \ldots & x_{k 2} \\
1 & x_{13} & x_{23} & \ldots & x_{k 3} \\
1 & x_{14} & x_{24} & \ldots & x_{k 4} \\
\vdots & \vdots & \vdots & \vdots & \vdots \\
1 & x_{1 n} & x_{2 n} & \ldots & x_{k n}
\end{array}\right) \times\left(\begin{array}{c}
a \\
b_{1} \\
b_{2} \\
b_{3} \\
\ldots \\
b_{k}
\end{array}\right)+\left(\begin{array}{c}
e_{1} \\
e_{2} \\
e_{3} \\
e_{4} \\
\ldots \\
e_{k}
\end{array}\right)
$$

onde $x_{j i}$ representa o valor da j-ésima variável da i-ésima observação, $a$ é a constante, $b$ coeficiente da variável $x$ e $e$ é o erro. Também é possível reescrever esta fórmula de forma resumida Eq.(22).

$$
y=X b+e
$$

A resolução deste modelo consiste na estimação dos parâmetros $a, b_{1}, b_{2}, \ldots, b_{k}$, que ocorre pelo método dos mínimos quadrados o qual é alcançado através da minimização da soma do quadrado dos erros $\sum_{i=1}^{n} e_{i}^{2}$.

No entanto, no presente trabalho para prever uma variável dependente $(Y)$, que corresponde a uma das medidas utilizadas, como as médias horárias das variáveis de: temperatura do ar, temperatura do solo e umidade relativa do ar ou as mensais de temperatura máxima do ar e umidade relativa do ar, foram utilizados como variáveis independentes $(X)$ as informações referentes à sua ocorrência, sendo elas horas ou meses conforme os dados utilizados. 


\subsection{Modelo adotado para predição de dados}

Neste estudo foram utilizados 677 registros de cada variável horária e 138 registros de cada variável mensal. Os dados foram pré-processados através da normalização, que alterou a escala real dos valores de entrada para um intervalo entre zero e um Eq. (23).

$$
x_{j}^{\text {norm }}=\frac{x_{j}-x_{j}^{\min }}{x_{j}^{\max }-x_{i}^{\min }}
$$

onde $x_{j}^{\text {norm }}$ representa a variável normalizada, $x_{j}$ a variável na posição $j, x_{j}^{\min }$ o menor valor entre as variáveis e $x_{j}^{\max } \mathrm{o}$ maior valor entre as variáveis.

Após a preparação dos dados, o conjunto de informações de cada variável foi dividido em duas partes, sendo $70 \%$ para treino/ajuste e $30 \%$ para validação.

Esse critério de divisão de dados foi adotado a fim de realizar uma comparação entre os valores observados e estimados pelas técnicas de predição.

Realizada a divisão dos conjuntos, os dados de treinamento foram divididos em entradas e alvos, para então, serem submetidos as RNAs, onde as redes tiveram por finalidade tentar predizer o valor do alvo $x_{(i+1)}$ para cada dado de entrada $x_{i}$, ou seja para cada uma das variáveis meteorológicas utilizadas neste estudo que foram submetidas as RNAs, o modelo proposto estima o próximo valor da respectiva variável. Após a fase de treinamento, os dados de validação foram submetidos às RNAs para predizer um passo a frente de cada dado submetido. Devido a normalização, os valores gerados pelas saídas das redes neurais se encontravam na escala entre zero a um. Por isso, aplicou-se a função que muda a escala dos valores para a escala real Eq. (24).

$$
x_{j}^{e c r}=\left(x_{j}^{\text {pre }} \times x_{j}^{\max }\right)-\left(x_{j}^{\text {pre }} \times x_{j}^{\min }\right)+x_{x}^{\min }
$$

onde $x_{j}^{e c r}$ representa a variável na escala real, $x_{j}^{\text {pre }}$ a variável predita pela rede, $x_{j}^{\max }$ o maior valor entre as variáveis e $x_{j}^{\min }$ o menor valor entre as variáveis.

\subsection{Avaliação do desempenho}

Para avaliar o desempenho das previsões geradas por cada modelo foram utilizadas medidas estatísticas, como o coeficiente de correlação de Pearson $(r)$ entre os dados preditos e os dados esperados, erro médio absoluto (EMA), raiz do erro médio quadrático (REMQ) e o erro médio percentual (EMP) (Fonseca et al., 2012; Deshmukh e Ghatol, 2010).

$$
\begin{gathered}
r=\frac{\frac{\sum_{j=1}^{n}\left(x_{j}-\bar{x}\right)\left(O_{j}-\bar{O}\right)}{N}}{\sqrt{\frac{\sum_{j=1}^{n}\left(x_{j}-\bar{x}\right)^{2}}{N} \times \sqrt{\frac{\sum_{j=1}^{n}\left(O_{j}-\bar{O}\right)^{2}}{N}}}} \\
E M A=\frac{\sum_{j=1}^{n}\left|O_{j}-x_{j}\right|}{n} \\
R E M Q=\sqrt{\frac{\sum_{j=1}^{n}\left(O_{j}-x_{j}\right)^{2}}{n}} \\
E M P=\frac{\sum_{j=1}^{n} \frac{\left|O_{j}-x_{j}\right|}{O_{j}} \times 100}{n}
\end{gathered}
$$

onde $n$ ou $N$, representa o número de dados utilizados, $O_{j}$ o valor observado, $x_{j}$ o valor predito pelas técnicas empregadas, $\overline{\mathrm{O}}$ a média dos dados observados e $\overline{\mathrm{x}}$ a média dos dados preditos.

Contudo, além das medidas já destacadas, também foram utilizadas para auxiliar a comparação entre os dados reais e os resultados apresentados pelos modelos as medidas de média (MED), máxima (MAX), mínima(MIN), desvio padrão (DESV PAD) dos dados reais e dos modelos de predição.

\subsection{Configurações adotadas}

Este estudo foi desenvolvido junto ao software MATLAB 7.4. A rede MLP foi implantada com duas camadas intermediárias e a RBF, como é de padrão, com apenas uma camada intermediária. As configurações das RNAs estão representadas na Tabela 1 .

\begin{tabular}{|c|c|c|c|c|c|}
\hline Modelos & $\mathrm{N}^{\mathrm{o}} \mathrm{NC} 1$ & $\mathrm{~N}^{\circ} \mathrm{NC} 2$ & F-PC1 & F-PC2 & NEP \\
\hline MLP & 10 & 5 & Tangente Hiperbólica & Logística & 4000 \\
\hline RBF & 1 a 30 & $\mathrm{X}$ & Gaussiana & $\mathrm{X}$ & 4000 \\
\hline
\end{tabular}

\section{Resultados e Discussão}

Para cada resultado apresentado por uma RNA foram realizadas 10 simulações que levaram cerca de 30 minutos cada, e calculado a média de cada dado predito para o respectivo período, ou seja calcula-se a média dos dados

Tabela 1 - Configurações adotadas pelas RNAs

onde: $\mathrm{N}^{\mathrm{o}} \mathrm{NC} 1$ é o número de neurônios da $1^{\mathrm{a}}$ camada intermediária, $\mathrm{N}^{\mathrm{o}} \mathrm{NC} 2$ é o número de neurônios da $2^{\mathrm{a}}$ camada intermediária, $\mathrm{F}-\mathrm{PC} 1$ é a função da $1^{\mathrm{a}}$ camada intermediária, F-PC2 é a função da $2^{\mathrm{a}}$ camada intermediária, NEP é o número de épocas do treinamento. 
horários ou mensais estimados em todas as simulações. Também foram aplicadas regressões lineares Múltiplas (RLM) na predição dos dados a fim de comparar os resultados. Feito isso, os resultados dos modelos foram analisados pelos índices de desempenho. Abaixo é apresentado os resultados obtidos.

\subsection{Resultados das predições das variáveis horárias - Paracambi}

A Tabela 2 apresenta as medidas de MED, MAX, MIN e DESV PAD dos dados reais, além dos erros obtidos pelos modelos na predição do conjunto de validação com 203 amostras de cada variável.

Pode-se observar que os modelos de RNAs apresentaram resultados superiores aos apresentados pelo modelo RLM (Tabela 2). É possível notar que o coeficiente de correlação $(r)$ entre os dados reais e os dados preditos de temperatura média do ar pelas RNAs MLP e RBF se encontram entre 0,92 a 0,93, demonstrando uma alta relação entre os dados estimados e os observados. Este fato pode ser visualizado na Figura 5, onde se observa que os dados reais e os preditos pelas técnicas utilizadas apresentam valores próximos.

No que tange os dados de temperatura média do ar, é possível observar que os EMP apresentados pela MLP e RBF não apresentaram diferença significativa $(0,05<\mathrm{p}$ valor), fato que não foi verificado quando comparado com o modelo RLM. Outro fator relevante na predição da temperatura média do ar são os resultados das medidas de erro apresentados pelos modelos, onde é possível verificar uma maior aptidão nas estimativas das RNAs, quando comparadas ao modelo RLM.
Para a variável de umidade relativa do ar, a sensibilidade das predições variou entre $91,27 \%$ a $91,39 \%$ para RBF e MLP, respectivamente. Comparando a correlação ( $r$ ) apresentada entre os dados reais e os preditos para umidade relativa do ar, verifica-se que as RNAs não apresentaram alteração em seus resultados, se mantendo em 0,91. Já a RLM apresentou valor de 0,76, sendo este inferior ao obtido pelas RNAs. Na Figura 6, pode-se observar que os dados preditos pelas redes mantiveram-se, em sua maioria, próximo dos dados reais, mostrando uma grande aptidão das RNAs na predição de umidade relativa do ar.

Na predição dos dados de temperatura média do solo, as RNAs mostraram desempenho superior as demais variáveis, tendo obtido menores erros e maior índice de correlação $(r)$. Este fato pode ser observado na Tabela 2. Analisando os resultados, constata-se que o EMP apresentado pela RNA MLP é, aproximadamente, $0,10 \%$ menor que o obtido pela RBF e, quando comparado ao erro obtido pelo modelo RLM, o modelo MLP apresentou valor quatro vezes menor.

Outro ponto de destaque apresentado pela MLP na predição dos dados de temperatura média do solo é o seu EMA, que é de $1,10^{\circ} \mathrm{C}$, valor esse inferior ao obtido pelo modelo de RLM. As demais medidas obtidas confirmam que os dados estimados pelas RNAs MLP e RBF foram consideravelmente próximos dos dados reais, possuindo poucas variações. Na Figura 7 é possível visualizar os dados reais e os preditos pelas RNAs para cada uma das 203 horas de temperatura média do solo.

Tabela 2 - Dados reais e resultados obtidos na predição das 203 horas. Índices: média (MED), máxima (MAX), mínima (MIN), desvio padrão (DESV PAD), coeficiente de correlação ( $r$ ), raiz do erro médio quadrático (REMQ), erro médio absoluto (EMA) e erro médio percentual (EMP).

\begin{tabular}{|c|c|c|c|c|c|c|c|c|c|}
\hline Dados Reais & MED & MAX & MIN & DESV PAD & & & & & \\
\hline Temperatura média do ar & 28,72 & 37,10 & 22,90 & 3,99 & & & & & \\
\hline Umidade relativa do ar & 63,31 & 93,00 & 31,00 & 16,68 & & & & & \\
\hline Temperatura média do solo & 33,23 & 41,20 & 27,50 & 3,90 & & & & & \\
\hline Dados Horários & Modelos & MED & MAX & MIN & DESV PAD & $(r)$ & REMQ & EMA & $\operatorname{EMP}(\%)$ \\
\hline \multirow[t]{3}{*}{ Temperatura média do ar } & MLP & 28,57 & 34,91 & 23,33 & 3,64 & 0,92 & 1,53 & 1,16 & 4,00 \\
\hline & $\mathrm{RBF}$ & 28,55 & 35,43 & 23,11 & 3,60 & 0,93 & 1,50 & 1,16 & 4,02 \\
\hline & RLM & 26,46 & 29,71 & 23,61 & 1,67 & 0,78 & 3,67 & 2,81 & 8,99 \\
\hline Dados Horários & Modelos & MED & MAX & MIN & DESV PAD & $(r)$ & REMQ & EMA & $\operatorname{EMP}(\%)$ \\
\hline \multirow[t]{3}{*}{ Umidade relativa do ar } & MLP & 63,91 & 93,36 & 33,97 & 15,93 & 0,91 & 6,87 & 5,17 & 8,73 \\
\hline & $\mathrm{RBF}$ & 64,82 & 88,24 & 40,67 & 16,90 & 0,91 & 7,14 & 5,12 & 8,61 \\
\hline & RLM & 75,25 & 86,16 & 62,77 & 6,44 & 0,76 & 17,27 & 13,97 & 28,81 \\
\hline Dados Horários & Modelos & MED & MAX & MIN & DESV PAD & $(r)$ & REMQ & EMA & $\operatorname{EMP}(\%)$ \\
\hline \multirow[t]{3}{*}{ Temperatura média do solo } & MLP & 32,75 & 38,25 & 27,13 & 3,74 & 0,94 & 1,45 & 1,10 & 3,19 \\
\hline & $\mathrm{RBF}$ & 32,74 & 37,76 & 27,49 & 3,21 & 0,94 & 1,51 & 1,22 & 3,49 \\
\hline & RLM & 28,26 & 31,11 & 25,77 & 1,47 & 0,70 & 5,83 & 5,00 & 14,27 \\
\hline
\end{tabular}




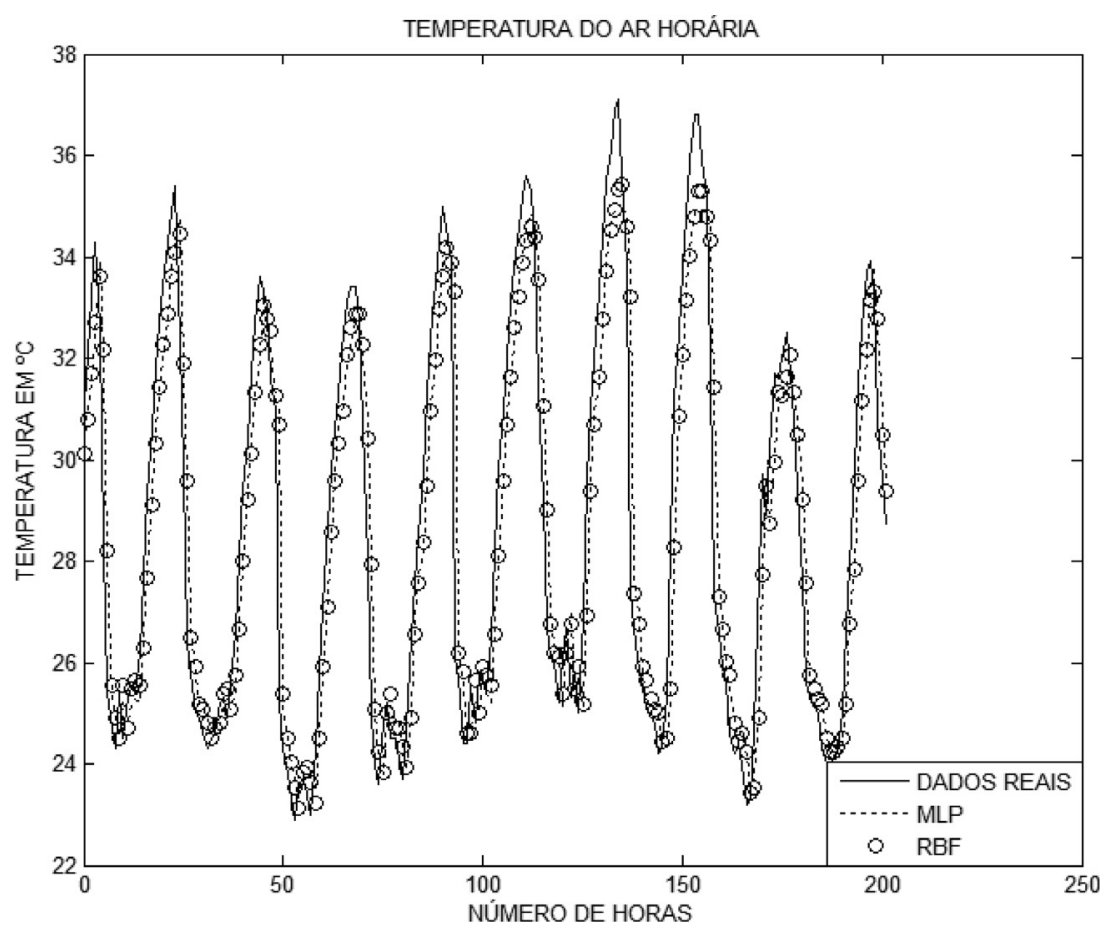

Figura 5 - Comparação das 203 horas de temperatura média do ar registrada e predita pelas RNAs MLP e RBF para Paracambi-RJ.

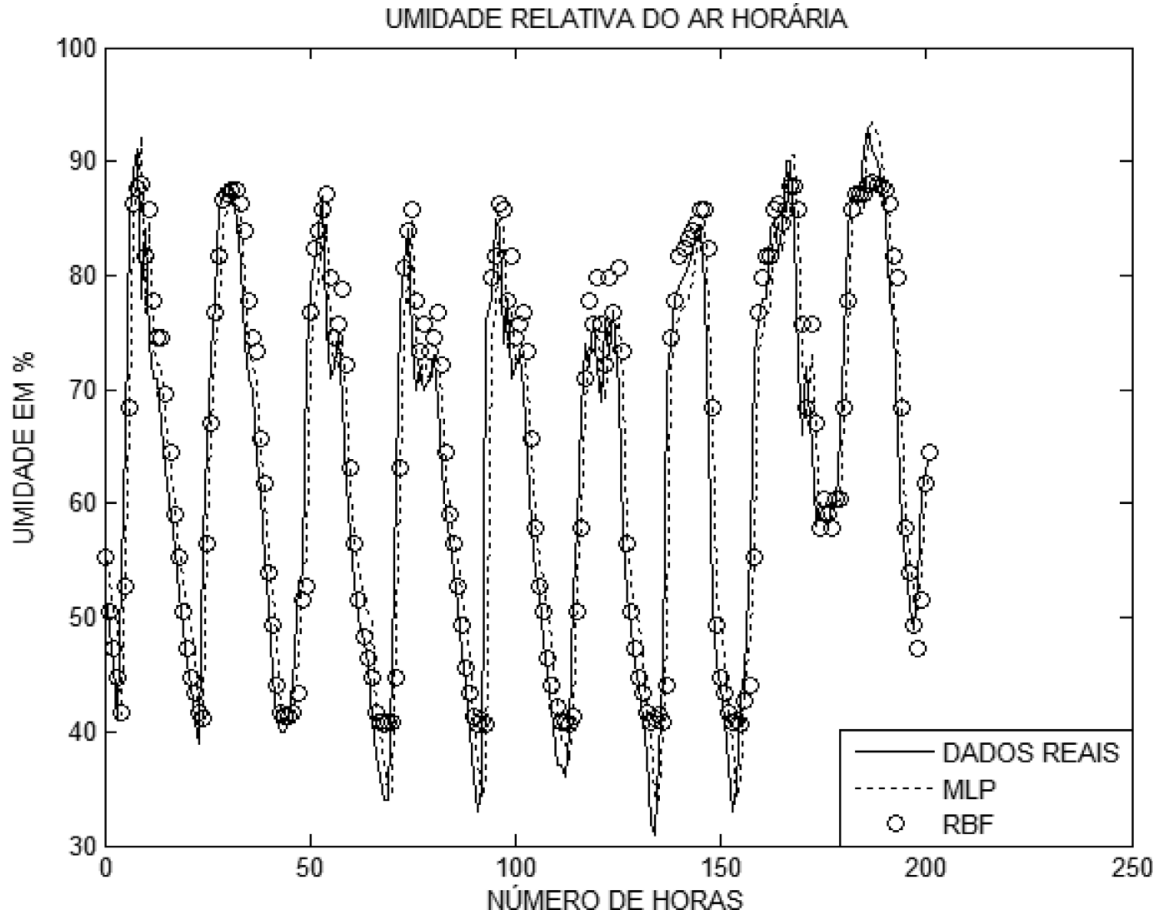

Figura 6 - Comparação das 203 horas de umidade relativa do ar registrada e predita pelas RNAs MLP e RBF para Paracambi-RJ.

\subsection{Resultados de predições das variáveis mensais - Paty do Alferes}

A Tabela 3 apresenta as medidas de MED, MAX, MIN e DESV PAD dos dados reais e os erros obtidos por cada modelo na predição do conjunto de validação, cerca de 41 meses.
Os resultados obtidos indicam que os modelos MLP e RBF foram mais precisos em suas predições, quando comparados com os resultados obtidos pelo modelo da RLM. $\mathrm{Na}$ predição da temperatura máxima do ar, os modelos MLP e RBF apresentaram um EMA entre $1,47^{\circ} \mathrm{C}$ e $1,63{ }^{\circ} \mathrm{C}$ e, um EMP entre 5,78\% e 5,24\%. 
Tabela 3 - Dados reais e resultados obtidos na predição dos 41 meses. Índices: média (MED), máxima (MAX), mínima (MIN), desvio padrão (DESV PAD), coeficiente de correlação (r), raiz do erro médio quadrático (REMQ), erro médio absoluto (EMA) e erro médio percentual (EMP).

\begin{tabular}{|c|c|c|c|c|c|c|c|c|c|}
\hline Dados Reais & MED & MAX & MIN & DESV PAD & & & & & \\
\hline Temperatura máxima do ar & 27,81 & 32,96 & 24,00 & 2,44 & & & & & \\
\hline Umidade relativa do ar & 78,45 & 85,41 & 66,00 & 4,23 & & & & & \\
\hline Dados mensais preditos & Modelos & MED & MAX & MIN & DESV PAD & $(r)$ & REMQ & EMA & $\operatorname{EMP}(\%)$ \\
\hline \multirow[t]{3}{*}{ Temperatura máxima do ar } & MLP & 27,58 & 29,32 & 25,39 & 1,20 & 0,61 & 1,89 & 1,47 & 5,24 \\
\hline & $\mathrm{RBF}$ & 27,73 & 30,53 & 25,74 & 1,45 & 0,57 & 2,03 & 1,63 & 5,78 \\
\hline & RLM & 27,56 & 28,62 & 26,65 & 0,61 & 0,17 & 2,49 & 2,01 & 7,20 \\
\hline Dados mensais preditos & Modelos & MED & MAX & MIN & DESV PAD & $(r)$ & REMQ & EMA & $\operatorname{EMP}(\%)$ \\
\hline \multirow[t]{3}{*}{ Umidade relativa do ar } & MLP & 78,89 & 82,60 & 72,66 & 2,30 & 0,68 & 3,21 & 2,53 & 3,31 \\
\hline & $\mathrm{RBF}$ & 78,84 & 82,59 & 72,68 & 2,43 & 0,63 & 3,37 & 2,66 & 3,47 \\
\hline & RLM & 78,62 & 80,97 & 76,78 & 1,25 & 0,29 & 4,05 & 3,25 & 4,25 \\
\hline
\end{tabular}

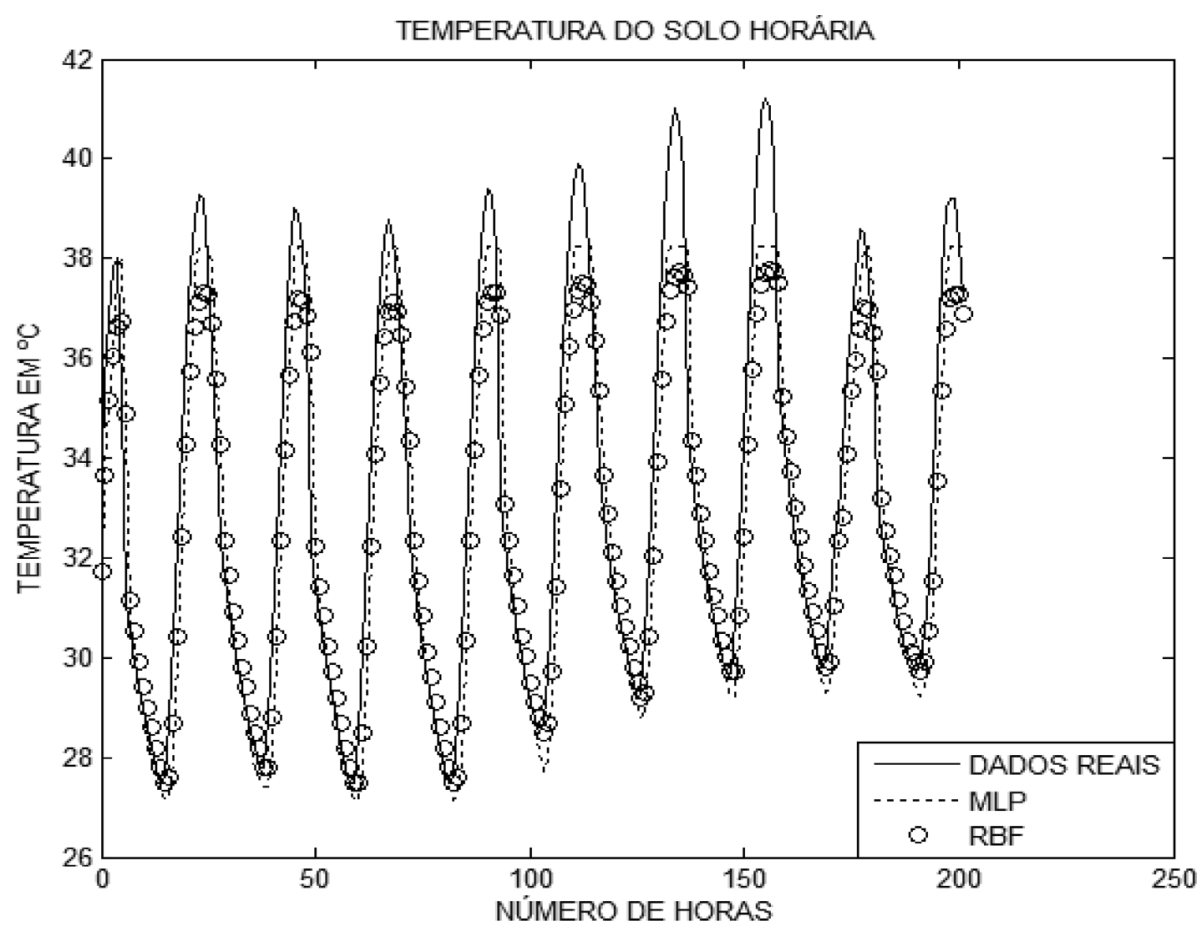

Figura 7 - Comparação das 203 horas de temperatura média do solo registrada e predita pelas RNAs MLP e RBF para Paracambi-RJ.

Entretanto, apesar da pequena diferença apresentada pelos modelos, observa-se que a RNA MLP obteve erros mais baixos, o que indica uma maior aptidão na predição de dados de temperatura máxima do ar mensal. Na Figura 8 verifica-se que apesar dos erros apresentados pelos modelos terem sido baixos, as RNAs MLP e RBF não conseguiram predizer os dados que apresentaram as medidas mais baixas e mais altas.

Para os dados de umidade relativa do ar é possível observar, por meio da Tabela 3, que os modelos neurais propostos apresentaram resultados similares. Entretanto, os mesmos foram superiores aos apresentados pela RLM.
Observa-se que o EMP obtido pelos modelos MLP e RBF se mantiveram entre $3,31 \%$ e 3,47\%, atribuindo uma precisão nas predições acima de $96 \%$. Outro fator importante pode ser visualizado comparando as medidas apresentadas pelos dados reais com os preditos, onde verifica-se que as RNAs obtiveram valores aproximados de MED e MAX, não conseguindo apresentar valores próximos de MIN e DESV PAD.

Também é possível verificar que o $(r)$ apresentado entre resultados preditos e esperados não ultrapassou 0,68 , o que infringe os demais resultados que caracterizaram uma boa predição. Na Figura 9 se nota que os modelos MLP e RBF não conseguiram reproduzir os dados mais baixos. 


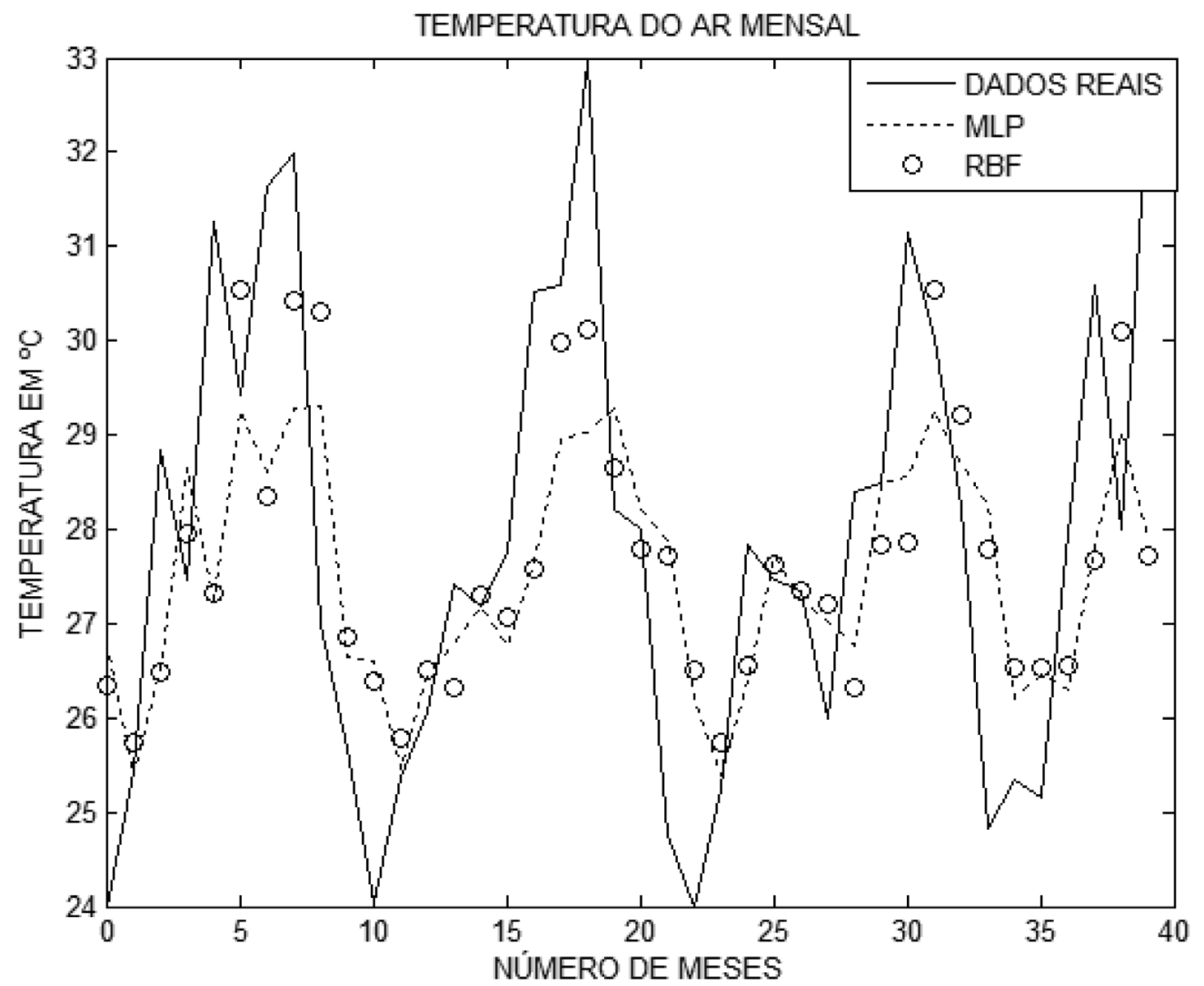

Figura 8 - Comparação dos 41 meses de temperatura máxima do ar registrada e predita pelas RNAs MLP e RBF para Paty do Alferes - RJ.

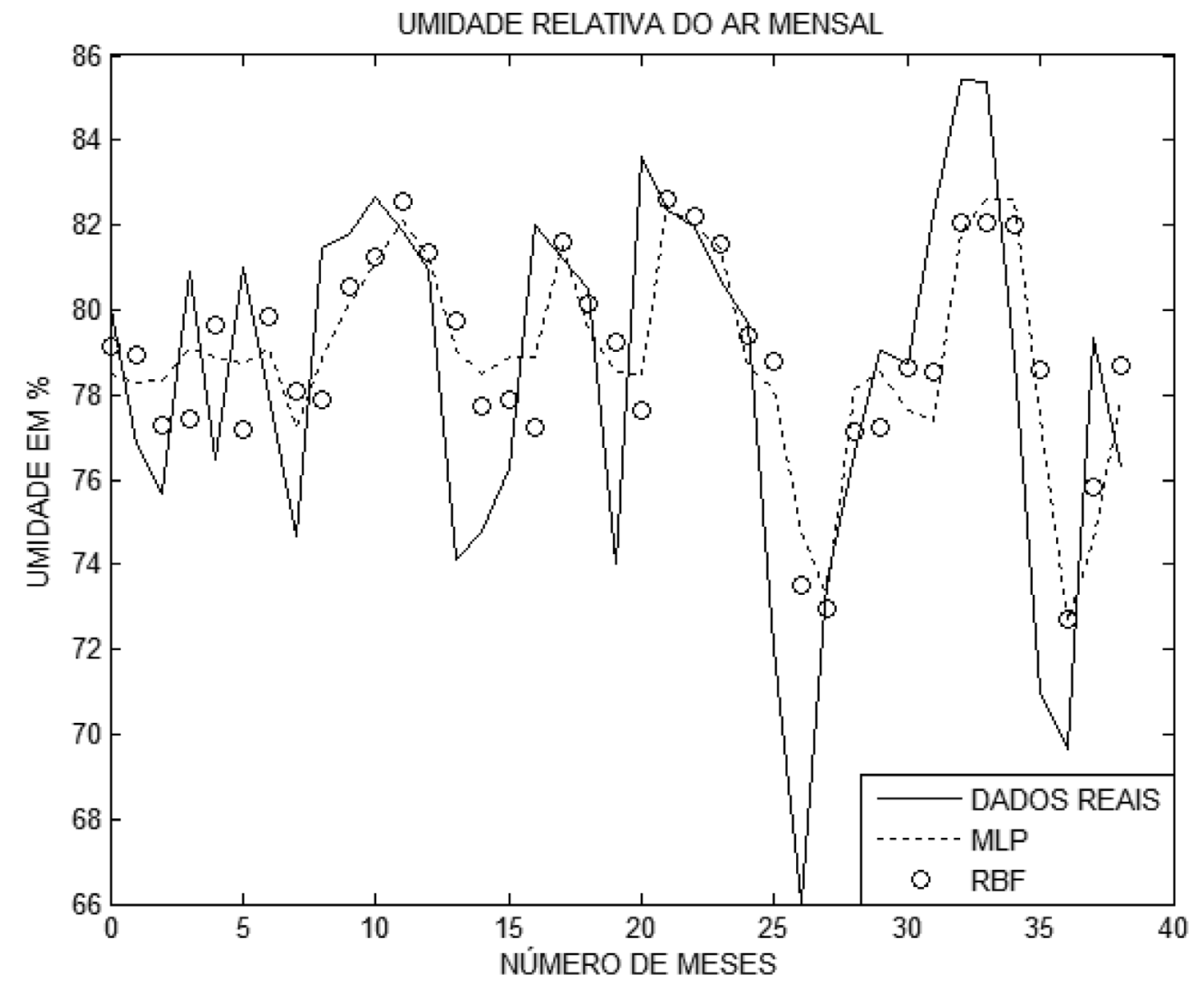

Figura 9 - Comparação dos 41 meses de umidade relativa do ar registrada e predita pelas RNAs MLP e RBF para Paty do Alferes - RJ. 


\section{Conclusões}

Através da análise dos resultados obtidos foi possível concluir que os modelos de RNAs: MLP e RBF apresentaram resultados satisfatórios, sendo superiores aos modelos de RLM. Contudo, é possível observar que os modelos tiveram predições mais apuradas para as variáveis horárias, que demonstraram maior relação entre os dados preditos e os observados por meio de suas correlações $(r)$. Isto pode ter sido influenciado pelo número de dados utilizados no treinamento das predições horárias que foram superiores aos dados utilizados no treinamento das predições mensais.

Além deste fato, observou-se que para a maioria das predições, tanto horárias quanto mensais, a RNA do tipo MLP apresentou os menores percentuais de erro médio (EMP), tendo tido um rendimento inferior apenas nas predições de umidade relativa do ar horária.

Entretanto, apesar de ser possível observar uma maior precisão nas previsões geradas pela RNA MLP, não se pode afirmar com exatidão qual dos modelos possui maior aptidão para predição de dados meteorológicos. Para tanto, se faz necessária a realização de outros trabalhos com a aplicação destas RNAs em outras regiões e com diferentes tipos de dados. Porém, pode-se afirmar que tais técnicas são ferramentas úteis para apoio da predição dos dados meteorológicos utilizados.

\section{Referências}

ALBARAKATI, N.; KECMAN, V. Fast Neural Network Algorithm For Solving Classification Tasks. IEEESoutheastcon Proceedings. p.1 - 8, 2013.

ALMOROX, J.; BENITO, M.; HONTORIA, C. Estimation Of Global Solar Radiation In Venezuela. Interciencia.v. 33, n. 4. p.280-283, 2008.

ASADUZZAMAN, MD.; AHMED, S. U.; KHAN, F. E.; SHAHJAHAN, MD.; MURASE, K. Making Use of Damped Noisy Gradient in Training Neural Network. IEEE - Neural Networks (IJCNN), The 2010 International Joint Conference on. p. 1- 5, 2010.

BOUGHRARA, H.; CHTOUROU, M.; AMAR, C. B. MLP Neural Network Based Face Recognition System Using Constructive Training Algorithm. IEEE - Multimedia Computing and Systems (ICMCS), International Conference on. p.233-238, 2012.

BRAGA, A. P.; CARVALHO, A. C. P. L. F.; LUDEMIR, T. B. Redes Neurais Artificiais - Teoria e Aplicações. Ed. 2, p. 225, 2012.

BUSTAMI, R.; BESSAIH, N.; BONG, C.; SUHAILI, S. Artificial Neural Network for Precipitation and Water Level Predictions of Bedup River. IAENG International Journal of Computer Science, vol. 34, p. 6, 2007.

CEPEA. PIB Agro CEPEA-USP/CNA - PIB do Agronegócio Dados de 1994 a 2011. Disponível em: http://www.cepea.esalq.usp.br/pib/. Acesso em agosto de 2013.

CEPED. CEPED - UFSC - Chuvas: por que o Brasil não consegue evitar essa tragédia. Disponível em : http://www.ceped.ufsc.br/noticias/chuvas-por-que-o-brasilnao-consegue-evitar-essa-tragedia. Acesso em março de 2014.

DESHMUKH, R. P.; GHATOL, A. A. Comparative Study of Temporal Neural Networks for Short Term Flood Forecasting. International Journal of Computer Applications, v. 5, n. 12. p. 24-28, 2010.

FALAYI, E.O.; ADEPITAN, J.O.; RABIU, A.B. Empirical models for the correlation of global solar radiation with meteorological data for Iseyin, Nigeria. International Journal of Physical Sciences, v. 3, n. 9. p. 210-216, 2008.

FONSECA, J. S.; MARTINS, G. A.; TOLEDO, G. L. Estatística Aplicada. Ed. 2, p. 267, 2012.

GODDARD, L.; MASON, S.J.; ZEBIAK, S.E.; ROPELEWSKI, C.F.; BASHER, R.; CANE, M.A. Current Approaches To Seasonal-To-Interannual Climate Predictions. International Journal Of Climatology. p. 1111- 1152, 2001.

GOIS, G.; SOUZA , J. L.; SILVA, P. R. T.; JÚNIOR, J. F. O. Caracterização Da Desertificação No Estado De Alagoas Utilizando Variáveis Climáticas. Revista Brasileira de Meteorologia - RBMET, v.20, n.3, p. 301-314, 2005.

GUARNIERI, R. A. Emprego de Redes Neurais Artificiais e Regressão Linear Múltipla no Refinamento das Previsões de Radiação Solar do Modelo ETA. 171 p. Dissertação (Programa de Pós-graduação em Meteorologia). Instituto Nacional de Pesquisas Espaciais-INPE, 2006. São José dos Campos-SP.

HALL, T., BROOKS, H.E.; DOSWELL, C.A. Precipitation Forecasting Using a Neural Network. Weather And Forecasting. 14:338-345, 1999.

HAYKIN, S. Redes Neurais Princípios e Pratica. Ed. 2, p. 902 , 2001.

HU, Y. H.; HWANG, J. Handbook of Neural Network Signal Processing. CRC Press, New York. p. 384, 2002.

LEMOS, R.M.A.; ALVES, A.P.P.; ASSIS, E.G.A.; CÔRTES, M.O.R.A. Dinâmica de Enchentes na Bacia Hidrográfica do Rio dos Macacos, Rj, Brasil. Congresso de Ecologia do Brasil, 9., 2009, São Lourenço. Anais... São Lourenço: Seb, P. 1-3

LITTA, A. J.; IDICULA, S. M.; MOHANTY, U. C. Artificial Neural Network Model In Prediction Of Meteorological Parameters During Premonsoon Thunderstorms. International Journal of Atmospheric Sciences, p. 14, 2013.

LYRA, G. B.; SOUZA, M. O.; VIOLA, D. N. Modelos Lineares Aplicados à Estimativa da Concentração do Material Particulado $\left(\mathrm{PM}_{10}\right)$ na Cidade do RIO DE JANEIRO, RJ. Revista Brasileira de Meteorologia-RBMET, v. 26, n.3, p. 392 - 400, 2011.

MAQSOOD, I.; KHAN, M. R.; ABRAHAM, A. An ensemble of neural networks for weather forecasting. Journal Neural Computing and Applications, vol. 13, n. 2, p. 112-122, 2004.

PALMER, T.N.; ANDERSON, D.L.T. The prospects for seasonal forecasting. Quarterly Journal of the Royal Meteorological Society. p.755-793, 1994.

ROLIM, J.; CATALÃO, J.; TEIXEIRA, J. Desenvolvimento de um interpelador espacial de dados Agrometeorológicos. Aplicação à Região Do Alentejo. III Congresso Nacional de Rega e Drenagem. Anais... Beja - Portugal .p.10, 2010.

SANTOS, C. C.; FILHO, A. J. P. Modelagem hidrológica urbana por meio de redes neurais artificiais: Uma aplicação para a 
bacia do rio Tamanduateí em São Paulo. Revista Brasileira de Meteorologia - RBMET, v.18, n.2, p.149-159, 2003.

SHAH, H. ; GHAZALI, R. Prediction of Earthquake Magnitude by an improved ABC-MLP. Developments in E-systems Engineering. 2011. P.6.

SONG, Q.; KASABOV, N. WDN-RBF: Weighted Data Normalization for Radial Basic Function Type Neural Networks. IEEE - Neural Networks, Proceedings. International Joint Conference on. p. 349 - 356, 2004.
SOUSA, N. M. N.; DANTAS, R. T.; LIMEIRA, R. C. Influência de Variáveis Meteorológicas Sobre a Incidência do Dengue, Meningite e Pneumônia em JOÃO PESSOA-PB. Revista Brasileira de Meteorologia-RBMET, v. 22, n.2, p. 183-192, 2007.

VIEIRA, H. J.; BACK, A. J.; LOPES, F.; MORAIS, H. Estimativa das Temperaturas Médias Diárias, Diurnas E Noturnas A Partir Das Temperaturas Horárias. Revista Brasileira de Meteorologia - RBMET, v. 27, n. 3, p. 329 - 336, 2012.

All the contents of this journal, except where otherwise noted, is licensed under a Creative Commons Attribution License CC-BY. 